\title{
DiÁSPORAS, IDENTIDADES Y MOVIMIENTOS POBLACIONALES NO TRANSNACIONALES
}

\author{
María del Carmen Peña Cuanda
}

Resumen: Este artículo es un esfuerzo por analizar a la luz de los diferentes debates teóricos que se han construido con relación a las diásporas y las identidades diaspóricas, un caso particular de movimiento poblacional interestatal (Chiapas, México) y voluntario (jóvenes que salen para realizar sus estudios universitarios) y que es el que me ocupa en el proceso de investigación que actualmente estoy desarrollando. El eje analítico fundamental es la noción de identidad, por lo que mi interés en este trabajo es construir un diálogo teórico entre mi investigación y los elementos que caracterizan a las diásporas.

Palabras clave: Identidad, diáspora, identidad diaspórica, desplazamientos de población intraestatal y de éxodo rural.

Enviado a dictamen: 22 de febrero de 2010 Aprobación: 18 de mayo de 2010

Revisiones: 1

María del Carmen Peña Cuanda, maestra en Psicología Social de Grupos e Instituciones por la Universidad Autónoma Metropolitana, Unidad Xochimilco (UAM-X). Docente de Tiempo Completo de la Universidad Intercultural de Chiapas. Estudiante de doctorado en Ciencias Sociales y Humanísticas, en el Centro de Estudios Superiores de México y Centroamérica (CESMECAUNICACH). Temas de investigación: Investigación, Metodología, Vinculación comunitaria, Educación intercultural, Comunicación, Psicología social, Identidades. Correo electrónico: mariacuanda@yahoo.com.mx.
Abstract: This article is an effort to analyze the several different theoretical debates surrounding the diasporas and the diasporic identities; in particular the interstate demographic migration (Chiapas, Mexico), voluntary in nature (young people driven out to pursue their advance studies) on which I occupy most of my time while in the process of the developing my current investigation. The fundamental analytic axis is the notion of identity, driving my interest to develop a theoretical dialogue between my investigation and the characteristic elements of the diasporas.

Key words: Identity, diaspora, diasporic identity, interstate demographic migration.

\section{Introducción}

E sta investigación ${ }^{1}$ se centra en el estudio de los procesos de resignificación identitaria que se tejen en el espacio universitario. Básicamente se centra en la reflexión personal y colectiva sobre los sentidos de pertenencia y en las posiciones identitarias significativas de los estudiantes de la Universidad Intercultural de Chiapas (UNICH). ${ }^{2}$

El interés central de la investigación radica en conocer cómo se transforma a partir de su llegada a la Universidad y a la Ciudad de San Cristóbal de Las 
Casas, la manera en que los sujetos se perciben a sí mismos (identidad personal y étnica) y cómo estas transformaciones son observables a partir de ciertas actitudes y comportamientos. Problematizar esta situación conduce a las preguntas de investigación, a saber: ¿A qué obedecen estas transformaciones? ¿Cómo son vividos los cambios por los jóvenes?, pero sobre todo al cambiar o resignificar sus identidades ¿Qué construcción de futuro de sí mismos está presente en ellos y cómo desde esta autopercepción orientan su acción?

Si bien la propuesta de investigación aún se encuentra en una fase temprana como para incluir los resultados del trabajo de campo en este debate, esto no exime de la posibilidad de problematizar la elaboración teórica de las diásporas como limitada a las poblaciones con movimiento transnacional, para pensar la construcción identitaria de los estudiantes, en su cualidad de sujetos en situación de movilidad intraestatal. Esto resulta posible porque se parte de tres presupuestos fundamentales que guían y organizan el sentido de este artículo:

a. La necesidad de pensar que los procesos de reconstrucción y resignificación identitaria que acontecen en las diásporas también ocurren en movimientos poblacionales no transnacionales.

b. Si bien no todo fenómeno de migración puede ser analizado, descrito o comprendido como diáspora, la teorización que sobre ella se ha generado aporta elementos para pensarlo en tanto que se fundamenta en la descripción de la experiencia, de la singularidad y del fenómeno en sí.

c. La aproximación metodológica del estudio de las diásporas da para estudiar otros fenómenos de asentamiento, movilidad o desplazamiento humano en las sociedades contemporáneas.

Por otro lado, la investigación tiene como uno de sus ejes teóricos y analíticos fundamentales la noción de identidad, por lo que mi interés en este artículo es construir un diálogo teórico entre mi propuesta de trabajo y los elementos que caracterizan a las diásporas y a las identidades diaspóricas. Por ello el cuestionamiento central del trabajo consiste en dilucidar si ¿Es la frontera geopolítica un elemento imprescindible para hablar de diáspora o de identidad diaspórica?

Por esto la hipótesis de trabajo que orienta las reflexiones aquí esbozadas es la siguiente:

Los movimientos o desplazamientos interregionales, locales, intraestatales, de éxodo rural o como quiera denominarse a la circulación de poblaciones (cambio de residencia) dentro de los límites establecidos desde la geopolítica por un estado-nación pueden desarrollar cualidades de las identidades diaspóricas.

A partir de estos elementos, la estructura y organización de las reflexiones que aquí presento es la siguiente: en la primera parte analizo las características de la diáspora y aportes metodológicos que me parecen pertinentes para pensar en las manifestaciones de un grupo que se ha movilizado dentro de las fronteras de un Estado-Nación, haciendo particular énfasis en la identidad diaspórica. Además, centro la discusión en que la limitación central de las elaboraciones teóricas ha sido pensar que las diásporas se fundamentan desde el transnacionalismo, es decir, se aborda el problema del Nacionalismo Metodológico.

Mientras que, en la segunda, trabajo específicamente con la noción de identidad y sus relaciones con la identidad de diáspora, centrando la discusión en los aportes que se han generado desde la teorización sobre diásporas para problematizar la situación y momento socio-histórico actual en la producción de subjetividades e identidades. Asimismo, muestro someramente lo que implica la "salida del hogar" (movilización o migración) en términos de la construcción identitaria y subjetiva de las personas. 
De las diásporas a los grupos movilizados dentro de un Estado-Nación.

Antes de comenzar con las reflexiones que me ocupan, considero necesario aclarar y tomarlo como punto de referencia básico para la discusión el hecho de que el grupo que yo estudio (jóvenes indígenas que han salido de sus lugares de origen y que son estudiantes de la Universidad Intercultural de Chiapas) no lo considero como una diáspora, si se parte del supuesto de que la diáspora implica un proceso de formación de comunidad o representa en sí misma una comunidad imaginada (Butler, 2001).

La población universitaria de la UNICH no es un grupo en sí, sino un conjunto de sujetos que asumen una misma posición (ser estudiantes, identidad ocupacional) durante un momento específico de su vida, lo cual les confiere un cierto proyecto común (terminar sus estudios, profesionalizarse, formarse), pero no por ello puede ser entendido como comunidad. ${ }^{4}$

Para este artículo es importante destacar que dentro de la población estudiantil indígena existen situaciones de movilidad social diferenciada, ubicando por lo menos a tres grupos:

a. El primero, son jóvenes que salieron de sus lugares de origen (comunidades o municipios indígenas) para poder realizar sus estudios universitarios o de nivel medio superior.

b. El segundo, los que nacieron en una comunidad indígena, pero que salieron de ella hace cinco años o más y no han regresado a vivir a estos espacios.

c. Asimismo, existe otro grupo que son hijos de migrantes indígenas, es decir, son jóvenes que nacieron en San Cristóbal de Las Casas, pero que sus padres o abuelos salieron de sus lugares de origen para asentarse en la ciudad.

Es significativo señalar que estos grupos continúan visitando con cierta frecuencia sus comunidades de origen o las comunidades de origen de sus padres y abuelos. No obstante, muchos de ellos refieren que no desean regresar a vivir a sus comunidades o que si lo hacen ya no se ven de manera permanente viviendo en ellas. No obstante, muchos de ellos tienen interés por "regresar" o "devolver" a sus comunidades algo que las beneficie en agradecimiento por el hecho de que ellos pudieron salir a estudiar y una forma de hacerlo es desarrollando sus investigaciones en estos espacios.

Por supuesto, ésta no es la condición en la que se encuentran todos los jóvenes, también hay varios que aunque sienten un apego a su cultura de origen, ${ }^{5}$ cuando hablan de sí mismos, plantean su deseo de vincularse y transformar la realidad de los espacios en los que actualmente se encuentran habitando. En estos casos la orientación de sus investigaciones se encamina hacia las nuevas comunidades de residencia, pero son la minoría, principalmente los casos de aquéllos que nacieron en una comunidad indígena y que salieron de ella hace cinco años o más. Por otro lado, de los jóvenes que nacieron en la ciudad más de la mitad desea trabajar en alguna comunidad indígena, pero de estos la mayoría desea hacerlo en una comunidad indígena que no es referida como la comunidad de origen de sus padres o abuelos.

Para este trabajo basta con señalar que estamos en presencia de un tipo particular de movilidad social, la migración intraestatal de éxodo rural que caracteriza a los sujetos de estudio y que será punto de partida para el análisis que se presenta a continuación.

El aporte interesante de la reflexión apunta al reconocimiento de ciertas características de las diásporas que dan para pensar movimientos de poblaciones no transnacionales en tanto fenómenos actuales e igualmente complejos, debido a que la amplitud, creciente movilidad o dispersión semántica, conceptual y disciplinaria del término (Brubaker, 2005; Butler, 2001), nos ayuda a pensar también algunas experiencias de minorías étnicas que viven en las ciudades, ya que la noción de diáspora en la actualidad 
es una designación metafórica que sirve para describir a diferentes categorías de personas.

(...) expression 'diaspora' is now being used (...) in a variety of new, but interesting and suggestive contexts (...) different caregories of people-'expatriates, expellees, political refugees, alien residents, inmigrants and ethnic and racial minorities (...) the term now designates a vast array of different peoples (Cohen, 1996: 514).

Igual de importante para este trabajo es que el pensamiento sobre la diáspora, su evolución y ruptura (Braziel y Mannur (2003), Brubaker (2005)), muestra un camino que han seguido en general muchas de las principales teorías y nociones del pensamiento social, por lo que ayuda a cuestionar posturas esencialistas o visiones cerradas porque parte de las condiciones actuales.

En otras palabras, metodológicamente ayuda a derribar estructuras de pensamiento ancladas en explicaciones de fenómenos de otras épocas o limitadas por los desarrollos científicos de sus momentos sociohistóricos. Además, desde esta perspectiva no implica un abandono de lo ya construido, sino un esfuerzo por resemantizar y resignificar desde la realidad contemporánea.

\section{Pensando diaspóricamente}

Un primer aporte sumamente interesante de la conceptualización de las diásporas en la actualidad es el que hace Brubaker (2005) cuando señala la imposibilidad de contar a los integrantes de una diáspora, por un lado, y con ello al indicar que la pertenencia a la misma es una cuestión de adscripción subjetiva y no una categoría social que se impone para clasificar a los sujetos, por el otro.

Esto nos sitúa bajo una perspectiva en la que la identidad en su función de conformación de un "nosotros" no es un atributo permanente de un grupo, es decir, la identidad es una práctica social que opera en los procesos de creación de sentido y pertenencia, y no es un atributo esencial que caracteriza a un grupo.

Esto nos ubica en un debate actual sobre el posicionamiento dualista que ha marcado profundamente las corrientes teóricas de la noción de Identidad (Dubar, 2002; Hall, 1999: Alcoff, 1989: Riquer, 1992).

Por un lado, está la postura esencialista, que básicamente remite a la conceptualización de la identidad como mismidad, aquello que nos da la cualidad de idéntico (de ahí el nombre), como sustancia inmutable o categoría natural compartida y transmitida, es el eidos (esencia) de las personas o grupos, por lo tanto algo que es permanente y atemporal. Todos estos son significados profundamente enraizados en el pensamiento filosófico y en el origen etimológico de la palabra.

La otra postura, más reciente, es definida como nominalismo (Dubar 2002), postura constructivista (Hall, 1996 y 1999, Giménez, 2000 y 2005I y 2005II, Castells, 1999), la definición posicional (Alcoff, 1989), cuestiona la identidad como una categoría dada o como un atributo de clasificación de individuos y grupos, plantea que la identidad está sometida a cambios y transformaciones, remite a los modos en que nos identificamos, la identidad construida desde una acción específica que es el proceso de identificación, se construye desde las formas de nombrar o nombres con que caracterizamos nuestras pertenencias, desde las posiciones enunciadas que se asumen en la representación de un yo o un nosotros, finalmente remite a una noción limítrofe, contradictoria, ambivalente o de frontera entre los social-cultural y lo individual o subjetivo.

Para muchos de estos autores la primera postura, es decir, la esencialista ha sido superada en términos conceptuales, pero se reconoce su eficacia discursiva y simbólica en muchos de los movimientos sociales que han emergido o cobrado 
fuerza en la época actual y que se han sustentado en la identidad como fundamento de movilización y cohesión social, ${ }^{6}$ partiendo de algún o algunos atributos que presuponen idénticos, permanentes o ahistóricos, aunque las movilizaciones de estos grupos están fundamentadas en procesos históricos de colonizaciones, exclusiones, discriminaciones, marginaciones, maltratos, negaciones, resistencias, confrontaciones, etcétera. No obstante, esta situación no derriba la postura nominalista o constructivista, pues la identidad retomada como bandera construida desde esencialismos en los movimientos sociales puede ser pensada como una posición asumida para la acción política (Alcoff, 1989) o como reunificación imaginaria que posiciona a los sujetos como actores políticos (Hall, 1999).

Estas posturas con relación a la identidad, en general, y a la identidad diaspórica, en particular, no es nada nuevo, pues el propio Barth (1969) ya lo había señalado al hablar de los grupos étnicos.

El aporte interesante de la teoría de la diáspora está en que se desterritorializan las identidades por la situación de no poderse afianzar a un solo territorio como marco referencial en la construcción simbólica, sino a múltiples. También implica que estamos en presencia de una identidad nunca fija y que se conforma de múltiples identidades en su seno (Butler, 2001) que la caracterizan por su profunda transformación permanente, por su definición siempre por contraste, gracias a la presencia del otro intrínseco.

Me parece que el fenómeno social que podemos observar en el caso de los jóvenes estudiantes que han salido de sus lugares de origen da cuenta perfectamente de estas situaciones, puesto que al comenzar a estudiarlos con mayor detenimiento encontramos que son múltiples los atravesamientos grupales, institucionales, territoriales, étnicos, culturales, etcétera, que convergen, y que en la práctica de ser estudiantes de la universidad dinamizan sus procesos de identificación.
Por ejemplo, los estudiantes ubicados en el tercer grupo, son hijos de padres o abuelos que salieron de sus territorios de origen, en algunos casos su padre es originario de una comunidad o municipio distinto al de su madre, en otros hay casos de desplazamientos territoriales por causas de expulsiones religiosas, en otros sus abuelos fueron reubicados de sus tierras de origen por algún interés político y sus padres decidieron migrar del nuevo territorio a la ciudad en búsqueda de mejores oportunidades, etcétera. Empero, el referente identitario no se afianza en un territorio específico, sino en el sentido imaginario de pertenencia a un lugar de origen común, al ancestro común y a una cultura compartida.

Otro aporte interesante de los estudios de la diáspora tiene que ver con el ámbito metodológico. La propuesta de Butler (2001: 195) indica cinco dimensiones que se deben considerar cuando se desarrolla un análisis de diáspora. Estas cinco dimensiones permiten solventar, por lo menos metodológicamente, las dificultades que actualmente se enfrentan frente a la dispersión conceptual y semántica de la noción, y son: razones por las cuales y condiciones de la dispersión; relaciones con el lugar de origen; relaciones con el lugar de residencia; interrelaciones internas entre las comunidades y personas que conforman la diáspora; $y$, estudio comparativo entre diferentes diásporas.

A partir de esta propuesta y en relación con mi investigación considero que son de destacar dos aspectos. En primer lugar, es muy interesante la apuesta por no desprenderse en ningún momento de la historia particular y circunstancias de la dispersión, aspecto que se sugiere con la primera dimensión. Este es un aporte útil y pertinente para estudiar cualquier fenómeno de movilidad social y no sólo los transnacionales, porque realizar el análisis y comprensión del fenómeno desde su especificidad, poniendo énfasis en la elucidación de la experiencia (única, singular e irrepetible) en el contexto contemporáneo debe ser una apuesta metodológica para la construcción de conocimientos 
que se sustenten en el estudio de la particularidad desde su especificidad.

Por otro lado, la segunda de las dimensiones remite a las relaciones con el lugar de origen y el autor refiere a que el constructo, construcción o representación del lugar de origen es esencial, porque funciona como base constitutiva de la identidad colectiva de la diáspora, ${ }^{7}$ aunque es importante señalar que este no remite a un espacio delimitado geopolíticamente (Butler, 2001: 204).

Desde esta perspectiva podemos ver la relación que se establece entre la identidad y la diáspora, pues la primera es un elemento constitutivo de la segunda y por lo tanto un elemento fundamental de análisis de sus procesos de conformación. Igual de relevantes son las relaciones con el lugar de habitación o de residencia en estos procesos de identificación, ya que entran en juego junto con las interacciones sostenidas (aunque sea imaginariamente) con los lugares de origen y con otros grupos que han salido de sus lugares de origen, con la sociedad mayoritaria ${ }^{8}$ del lugar de residencia.

Básicamente lo que me interesa destacar es que estos procesos de reconfiguración de las identidades entran en juego en poblaciones que se han desplazado de sus lugares de origen y que las nuevas identificaciones se van tejiendo desde las contradicciones y múltiples atravesamientos que se desarrollan tanto en las relaciones sostenidas con los lugares de origen, como con los de residencia actual y por el contacto con otras poblaciones que también se han movilizado.

En el caso de los estudiantes de la UNICH claramente podemos observar que hay orígenes diversos, es decir, conectan comunidades múltiples y culturas multilocales (Clifford, 1997), que se van generando relaciones entre jóvenes que vienen de otros lugares del estado, pero también con jóvenes que han nacido en San Cristóbal de Las casas y cuyos padres tienen historias diversas, algunos son migrantes de primera o segunda generación, otros provienen de familias que siempre han vivido en el lugar, y otros son hijos de extranjeros o de mexicanos que provienen de otros lugares de la república mexicana. Y aunque no todos estos tipos de población puedan encontrarse en la universidad, son grupos con los que pueden entrar en contacto e influir en el desarrollo de estos procesos.

Al trabajar con estos estudiantes es posible observar que a su llegada a la universidad, espacio que se encuentra inserto en una ciudad en la que tienen contacto con culturas diferentes a la de ellos, comienzan a tener cambios en sus formas de comportarse, de expresarse, de vestir, de hablar, de relacionarse, etcétera. Estos cambios son importantes para ellos y se preguntan por los motivos y significados de los cambios que están observando en sí mismos y en los "otros" (sus compañeros y compañeras).

Esta situación de transformación en las identidades no sólo nos remite a los casos de los jóvenes que acaban de cambiar su lugar de residencia con motivo de poder desarrollar sus estudios en la universidad, sino también en aquellos que han nacido o crecido en la ciudad pero cuyos padres o abuelos son originarios de alguna comunidad indígena, y que al entrar en la universidad se encuentran en un espacio en el que, al menos en el plano discursivo, se valora su origen, muchas veces negado por ellos mismos o por sus padres para evitar la discriminación y el rechazo en otros espacios de la ciudad.

Esto demuestra que aunque hay situaciones de dispersión de población (aludiendo a una de las concepciones básicas y tradicionales de la diáspora) que si bien pueden no ser integradas como diáspora, pueden presentar dimensiones diaspóricas en sus prácticas y culturas.

Los jóvenes de la UNICH comparten lo que Clifford J. (1997: 302) comenta como formas diaspóricas de “añoranza, memoria y (des)identificación". Aunque es necesario considerar que estos jóvenes a la par de los procesos de (des)identificación están desarrollando procesos de revaloración del vínculo y por ello entran en un proceso de (re)identificación, lo que 
puede apreciarse en sus intereses por desarrollar investigaciones relacionadas con las culturas de los pueblos originarios y en territorios indígenas. Estos procesos acontecen simultáneamente y de manera tensa, contradictoria y ambivalente.

Por otro lado, una de las principales limitaciones que encuentro en el estudio de las diásporas es que la identidad cuestionada es la nacional (o étnico-nacional) ¿y qué hay entonces de las otras identidades colectivas que se juegan en los procesos de movilidad social? ¿Qué pasa con estas otras identidades que ahora cobran fuerza en los procesos de integración y conformación social? ¿Qué hay de los grupos que no retoman la identidad nacional como referente significativo en el proceso de identificación?

No podemos dejar de pensar en dos elementos de gran importancia con relación a esta cuestión: en principio, el hecho de que las identidades nacionales son (o fueron) proyectos políticos que se han reconfigurado a partir de la propia reconfiguración global del planeta (Habermas, 1996; Hobsbawn, 1995; Adamson y Demetriou, 2007). En segundo lugar, al lado de la idea de homogeneización que produce la globalización, se acentúa la idea de la conformación, reconocimiento y acentuación de la diversidad, pues en el proyecto global múltiples culturas están siendo sincretizadas ${ }^{9}$ de un modo complejo (Cohen, 1996), y esto se puede ver y comprender a través del estudio de la experiencia de las diásporas en su sentido amplio. En otras palabras: las diásporas ayudan a pensar fenómenos sociales e identitarios desarrollados en las nuevas condiciones globales.

El discurso de la diáspora (...) Anda libre por el mundo, debido a razones que se relacionan con la descolonización, la inmigración creciente, las comunicaciones globales y el transporte: toda una variedad de fenómenos que estimulan los apegos multilocales, la residencia y el viaje dentro de las naciones y a través de ellas. (Clifford, 1997: 305)
Enfatizo el pensamiento de la diáspora en su sentido amplio básicamente para señalar una de sus principales debilidades en su construcción teórica, a saber: el limitar gran parte de sus análisis y propuestas a los movimientos poblacionales que se remiten a lo nacional.

Una de las principales críticas en este sentido es la que construyen Wimmer y Glick Schiller (2002) cuando señalan que el Nacionalismo metodológico ha sido una forma de invisibilizar el panorama social o de construirlo de forma naturalizada a partir del proceso de construcción y formación de los Estados-Nación. Creo que este es un problema del que no se ha desprendido el estudio de las diásporas, ya que en muchos casos sigue anclado en el estudio del fenómeno desde el nacionalismo y el transnacionalismo.

Estas autoras reconocen que los movimientos internos de los estados-nación carecen de importancia en muchas de las elaboraciones teóricas contemporáneas en la medida en que no cuestionan el orden nacional y su proyecto.

Si hay desplazamiento de la nación (entendida desde su etimología de nacido en) o mejor dicho del lugar de origen (territorios étnicos) pero no del estado-nación, entonces no hay una pérdida de lo nacional en un sentido político, pero ¿Qué hay del orden simbólico, personal, comunitario y subjetivo? ¿Cómo podemos denominar estas experiencias? ¿Cómo definimos y se autodefinen estos sujetos? ¿Caben los discursos del nacionalismo plural (Smith, 1991)?

Si se parte del supuesto que ser inmigrante o emigrante es ser nacional o no nacional (Sayad, 2009), encontramos las limitaciones del problema y muchos desplazamientos de población se dejan de ver en los análisis. No obstante, haciendo un esfuerzo por vislumbrar de manera compleja el fenómeno migratorio (pensándolo como movilización o cambio de residencia) entonces puede comprenderse que debe ser estudiado como proceso y no debe ser minimizado en importancia si sale de los discursos "relevantes" construidos desde el nacionalismo metodológico. 
No me atrevo a afirmar que el argumento de Wimmer y Glick Schiller (2002) sea una propuesta para ver más allá de los límites que la formación social moderna (pensada como nación-estado-sociedad), pero por lo menos sí se le puede reconocer como una preocupación por pensar eso que puede no ser importante desde las valoraciones hegemónicas, lo impensado o invisibilizado desde el pensamiento nacionalista.

En el caso de los jóvenes con los que estoy trabajando, encontramos que al ser indígenas se ubican como minorías autóctonas de los estados-nación que históricamente han desarrollado procesos de resistencia o lucha por el reclamo de su afirmación como sujetos autónomos, es decir, reclamos contra una hegemonía nacional opresiva (Clifford, 1997) que en su movilidad dentro del espacio nacional, se van apropiando de escenarios que los dotan de visibilidad para autentificar las demandas por su reconocimiento.

La universidad en la que estudian es un espacio que muestra la institucionalización de políticas culturales públicas que han posibilitado dicho reconocimiento, todavía circunscrito al ámbito nacional. En búsqueda de la armonía posible y porque no se irrumpa en el ámbito del proyecto político amplio del estado-nación mexicano, es decir, políticas culturales que promueven o por lo menos proclaman un nacionalismo plural (Smith, 1991).

En este sentido podemos afirmar que los estudiantes no luchan en estricto sentido desde un reclamo, ya que asumen las normas del estado-nación hegemónico a través de sus instituciones. Sin embargo, esto no significa una pasividad e indiferencia, podemos sugerir que la dispersión de estas poblaciones también se sustenta en el reconocimiento personal, público y colectivo de su lugar como ciudadanos de primera.

Inmersos en un discurso institucional (UNICH) en donde las particularidades culturales de sus lugares de origen son importantes (y convenientes), les posibilitan espacios de relación y los dotan como actores políticos, agentes y promotores del cambio. De manera simultánea, contradictoria y generando ciertas tensiones, también se enfrentan a prácticas de residencia que transforman sus esquemas de significación aprendidos en el hogar ${ }^{10}$ (Schutz, 1974) y desde las culturas.

A estos procesos se suma la ambivalencia que se genera desde la situación de residir en un lugar, Aquí, en donde se generan lazos de solidaridad o vínculos sociales con los que también están; mientras que se acentúan de distintas maneras los lazos con el Allá, que no implica necesariamente un solo lugar. Recuérdense los tres elementos que destaca Clifford, para comprender y definir a las diásporas como fenómenos sociales:

(...) se la entiende como una práctica de residencia (...), como una negativa ambivalente o un aplazamiento indefinido del regreso y como un transnacionalismo positivo (...) (Clifford, 1997: 329).

Salvo el tercer elemento, discutido previamente, e inexistente en los sujetos de estudio de mi investigación, podemos tomar los otros dos elementos para caracterizarlos. Al hablar de práctica de residencia es importante aclarar que no necesariamente todos tengan las mismas prácticas, pero sí que pueden desarrollar algunas similares. Al hablar de la negativa ambivalente podemos encontrarla cuando al preguntarles a los jóvenes sobre sus intereses de investigación y futuro la mayoría manifiesta un marcado interés por estudiar y conocer sus culturas o las de sus padres y abuelos, pero pocos son los que manifiestan una intención real por regresar a vivir a sus lugares de origen.

La confrontación mencionada con múltiples espacios y diferencias, los lleva a sentir (según ellos lo nombran) un "sin sentido", poca claridad sobre sus representaciones y sus sentidos de pertenencia. Esto habla de un momento de quiebre en sus historias que los pone en movimiento y dinamiza sus procesos de identificación.

Y esto nos devuelve a la noción de identidad como práctica social, pero también como identificaciones, 
como procesos no predeterminados que son reinventados en forma permanente. Con lo que podemos afirmar que la identidad diaspórica es un referente y fundamento conceptual para pensar en la actualidad a muchas de las identidades existentes (nombradas y practicadas), todas aquellas que se puedan caracterizar como identidades híbridas contemporáneas (Clifford, 1997), aquellas que emergen y se reestructuran del inevitable contacto con los "otros", con lo "diferente", lo "extraño", lo "ajeno".

\section{Identidades movilizadas... Identidades "diaspóricas"}

Desde las reflexiones del apartado anterior y con relación a las preguntas centrales que guían este artículo puede verse mi preocupación por las relaciones entre la diáspora y la identidad, por ello en este apartado muchos de los elementos que retomo tienen que ver con la forma en que los aportes teóricos que se han desarrollado desde la diáspora son útiles para pensar las identidades de las personas que han cambiado sus lugares de residencia con relación a sus lugares de origen, es decir, me centro en el análisis de la identidad diaspórica como elemento que igualmente da para pensar los procesos de movilización no transnacionales.

Lejos estamos de encontrar consenso alguno en la definición de identidad, mucho menos si se retoma la misma desde un enfoque multidisciplinario. No obstante, en los diferentes textos podemos ubicar un consenso: que el problema y objeto de conocimiento de la identidad sólo cobra sentido en la época moderna, no importa cómo se reconozca y se nombre a esta, no importa tampoco desde qué campo de conocimiento se esté analizando el problema. La identidad, a pesar de la confusión y maraña de significaciones y formas de interpretarla, por acuerdo de muchos teóricos, es importante en las ciencias a partir de la relación histórica que guarda con las características de la sociedad actual.
En este mismo sentido, el estudio de las identidades y de las diásporas (o mejor dicho de las identidades diaspóricas) hoy en día remite al diálogo y cuestionamiento de los nacionalismos, en tanto que estos últimos remiten al campo de la universalidad (o por lo menos de la "Comunidad Imaginada" (Anderson, 1983) y los ideales teóricos que la sustentan) y las primeras lo hacen más a la diversidad e hibridez cosmopolita (Anderson, 1998).

El pensamiento nacionalista, en términos políticos (cuando menos), se valida como una forma de vida social cuando pasan dos cosas: por un lado, son visibles las instituciones y prácticas sociales especializadas; y por el otro, cuando el mundo es entendido como uno, sin importar cuantos sistemas políticos, sociales, religiosos, lingüísticos, culturales, económicos diferentes contenga, porque hay una actividad común: La Política. (Anderson, 1998).

Por su parte Hobsbawn (1995) caracteriza al siglo XX como el de mayor crecimiento y transformación social que se ha observado durante un periodo similar, transformaciones que han conducido a profundas crisis en todas las formas de organización social. Como resultado de estos resquebrajamientos de las estructuras sociales, la identidad se puso al centro como una posibilidad de marcar la diferencia frente a la homogeneidad aplastante que promovía el sistema económico y los nacionalismos (o identidades nacionales) que se forjaron desde el siglo pasado y que sirvieron a este propósito como lo comenta Akzin (1964: 26-27).

Otra tendencia significativa en la literatura del siglo pasado, es la que fuertemente afirma la superioridad de la nacionalidad propia del autor (o su nacionalidad favorita) sobre todas las demás o cuando menos sobre algunas otras. La conciencia de la propia pertenencia a una nacionalidad determinada y una intencionada autoidentificación con ella (la llamada consciencia nacional) quedan intensificadas en esta tendencia (...).

130

Revista LiminaR. Estudios sociales y humanísticos, año 8, vol. VIII, núm. 1, junio de 2010, San Cristóbal de Las Casas, Chiapas, México. ISSN: 1665-8027 
Destaco esta situación porque justamente las identidades (incluidas las diaspóricas) atentan y contradicen este presupuesto político que pone en cuestión a los Estados-Nación como unidades políticas.

Así, la proliferación de la manifestación de las diferencias lo que ha hecho es transformar el pensamiento sobre las sociedades que ha imperado hasta bastante entrado el siglo XX. Ser distinto, hoy por hoy, se erige como bandera identitaria para realizar la composición del mundo, para situarse como actor político en la realidad.

Para problematizar sobre los aspectos de las diásporas y de las identidades se debe partir de premisas ontológicas y epistemológicas que permitan construir reflexión en los bordes de la modernidad (o más bien, considerándola en su complejidad real).

Para ello Hall (1999) propone situar a la identidad como una producción que nunca está completa y que se construye dentro de la representación ${ }^{11}$. Por esto, la identidad es situacional (lugar de emergencia) y contextual (histórico-social), porque siempre se encuentra en relación directa al lugar específico de su producción y al momento histórico en que es posible su emergencia.

Las formas en que un sujeto se identifica están relacionadas de manera directa (aunque no causal, como lo demuestra la diversidad reconocida) con el lugar en el que emergen, en el caso mi investigación la UNICH es el espacio que importa en los procesos de construcción de significado. Por otro lado, la identidad se produce siempre desde unas condiciones sociohistóricas específicas, que son aquellas que caracterizan al momento actual. Para Dubar (2002: 24)

El subimiento de nuevas formas de individualidad se considera aquí como el resultado, ni voluntario ni programado, de procesos que modifican los modos de identificación de los individuos como consecuencia de grandes transformaciones en la organización económica, política y simbólica de las relaciones sociales.
Esta postura se relaciona con el reconocimiento de que el momento actual tiene una forma específica de construir subjetividad que permite hablar de identidades híbridas contemporáneas (Clifford, 1997).

Las identidades diaspóricas son consideradas como formaciones identitarias que se construyen desde lo híbrido, el sincretismo, partiendo del reconocimiento necesario de la heterogeneidad y diversidad (Brubaker, 2005; Hall, 1999). Por esto, todo el desarrollo conceptual que se ha generado desde el análisis de las diásporas ha contribuido considerablemente para pensar en general las identidades en la modernidad.

Por otra parte, si se analizan los tres elementos que reconoce Brubaker (2005) para definir y diferenciar la diáspora de otros fenómenos sociales: 1. Dispersión espacial; 2. Orientación al territorio de origen; y 3. Fronteras o delimitación mantenida dentro del territorio de habitación actual; encontramos que el tercero remite a una frontera social (no política o territorial) que permite al grupo diferenciarse del resto de la sociedad, en este caso de la sociedad de residencia. En este sentido la noción de frontera sirve como límite para marcar la diferencia y afirmar determinadas relaciones que son reconocidas social y culturalmente por un grupo (Fábregas, 1984).

Esta concepción de frontera no es nueva y nos remite a la historia del término. La frontera en la actualidad es una noción que está cargada fuerte y profundamente de connotaciones políticas y que sirve para definir la línea que separa a las comunidades que jurídicamente se encuentran integradas bajo la forma de EstadosNación (geopolítica). No obstante, rastreando el origen del término nos encontramos con el otro sentido que resulta central para este trabajo: pensar a la frontera como construcción imaginaria de la diferencia, y por lo tanto de la similitud, lo que nos remite a la Edad Media, época en la que la frontera era concebida como una "categoría mental" (Mitre, 1997).

La identidad puede ser pensada como límite, como proceso social de delimitación de diferencias y 
similitudes o sentidos de pertenencia, y en este sentido estoy ubicando uno de los posibles sentidos que adquiere la noción de frontera. Por ejemplo, D’Andrea (2005: 62) comenta: Sancionar una pertenencia significa al mismo tiempo delimitar una diversidad: reconocer y circunscribir un espacio compartido, trazar una frontera, definir un afuera.

Esto da para pensar no sólo en las diásporas como comunidades o grupos que desarrollan identidades creativas y nuevas, sino igualmente en los procesos subjetivos de significación e identificación que se desarrollan en los sujetos que se encuentran en situaciones de dispersión poblacional no transnacional. Es decir, en la generación de procesos de "reajuste simbólico e imaginario" que se hacen inevitables en una situación de ruptura con lo cotidiano-conocido, con el hogar.

Pensar la vuelta al hogar con Schutz (1974) da para analizar las identificaciones que se desarrollan con el "hogar" (el lugar de origen) una vez que se está fuera de él, como sucede con muchos de los estudiantes de la universidad.

En primer lugar, presupone que el hogar significa una cosa para el que nunca lo ha abandonado, otra para quien habita lejos de él y otra para quien retorna. El marco referencial conocido permanece en el hogar, pero no en aquel (aquella) que deja de habitarlo, pues el sistema de significatividades (Schutz, 1974) conocido, compartido y hasta cierto punto previsible dentro de sus límites de modificación inesperada y transformable (como describe el habitus de Bourdieu, 1980), se reestructura en la persona que sale. En pocas palabras, en los que han dejado el hogar cambia el sistema de significaciones compartidas, sin importar si se cruza o no una frontera geopolítica, aunque sean procesos distintos y en distintos niveles o grados. Y esto que ocurre a nivel personal es un interés central de mi investigación. Un(a) joven que ha salido de su hogar tendrá una concepción del mismo y de sí mismo, primero en la distancia y, después, a su regreso, si es que éste acontece.
A diferencia de lo que puede reconocerse en las diásporas, en el caso de los jóvenes universitarios tienen múltiples retornos a sus hogares, aunque ninguno de ellos definitivo (por lo menos no durante el tiempo que duran sus estudios). Pero siguiendo con Schutz (1974) el regreso implica regresar como un desconocido dadas estas transformaciones inevitables en lo subjetivo que se producen por la movilidad o migración.

Entonces ien dónde encuentran ahora un lugar de referencia significativa cuando no son más del lugar en el que nacieron y crecieron, pero tampoco lo son del lugar en el que habitan? Esto produce una sensación de desposeimiento o desubicación que implica la partida del hogar. No obstante, la situación de estarfuera desarrolla distintas formas de vinculación con el hogar (ya sea imaginario o real).

Por supuesto la movilización de sus lugares de origen es una característica compartida por muchos estudiantes de la UNICH. Empero, no debemos dejar de pensar en estos otros jóvenes que nacieron en la ciudad de San Cristóbal de Las Casas y que son hijos, nietos o nacidos en alguna localidad indígena y que también hay en ellos una construcción (aunque sea imaginaria) del lugar de origen u hogar de los padres o abuelos y que desarrolla distintas cualidades en el vínculo y en su identidad.

Por ejemplo, encontramos jóvenes que son migrantes de segunda o tercera generación y que son hijos de expulsados religiosos y se autodefinen como indígenas, hablen o no la lengua originaria, que desean estudiar y conocer más sobre el grupo étnico de sus padres o abuelos, sin interesarse por regresar al territorio de origen de los mismos; hay otros que reconfiguran sus creencias religiosas tradicionales cuestionando las de sus propios padres y declarándose ateos y generando simpatías con otro tipo de agrupaciones juveniles (grupos de baile moderno, estudiantinas, hippies, etcétera); o hay algunos que cuestionan las decisiones de sus padres y abuelos y reivindican la cultura original y desde su posición de estudiantes desean estudiarla y promoverla. ${ }^{12}$ 
Volviendo al tema, la situación de movilidad social, permite a su vez hablar de cambios culturales continuos y constantes, que a su vez se reflejan en la propia identidad, porque una persona que se enfrenta o convive de manera directa con personas de otras culturas, puede cambiar las construcciones de su propia identidad para reafirmar su pertenencia a su grupo, al tiempo que para integrarse a nuevos grupos de pertenencia. De hecho García-Canclini (2000) sostiene la idea de que esta situación de contacto entre diversas etnias que él caracteriza como "hibridación intercultural", fomenta el acceso a muchos y muy variados bienes simbólicos y a experiencias nuevas para los sujetos que se inscriben en relaciones cosmopolitas:

\section{(...) creemos que se están generando nuevas modalidades de vínculo y de pertenencia, (...) una nueva subjetividad compartida socialmente, en suma, nuevas formas de construcción identitaria. La educación cumple un rol esencial en tales procesos al ampliar el "mundo de vida" de los jóvenes (...) por ello creemos que es esencial para la educación que va en el sentido del cambio y del respeto de las especificidades, la comprensión de estas nuevas modalidades o formas de construcción identitaria (Pintor, 2000: 110-111).}

Bauman (2005) analiza y comenta, al igual que lo hace Maffesoli (1988), la falta de proyecto que existe en muchos de los sujetos (individuales y colectivos) de la modernidad y su relación con el sentido de pertenencia. La fugacidad de las relaciones y los espacios de convivencia dificultan la construcción de un sentido más profundo, por ejemplo, en la actualidad las condiciones laborales cada vez hace más común la situación de la contratación por fuera (free lance) o por tiempo determinado (contratos que se hacen o no renovables según a conveniencia de los empleadores) y con esta situación las relaciones laborales también se delimitan temporalmente.
Además está presente el fenómeno de la ofimática en donde todos los procesos de oficina se realizan en una relación entre el trabajador y los aparatos tecnológicos, en este sentido es mediada, mejor dicho mediatizada, la relación.

Además, cualquier tipo de movilidad poblacional produce efectos tanto en las comunidades de migrantes, como en las comunidades de origen de los mismos. La cultura, las etnias, las identidades, se reconfiguran mostrando una faceta tanto positiva (formas de integración y solidaridad) como negativa (nuevos conflictos sociales entre grupos y al interior de los mismos). Las identidades diaspóricas manifiestan y evidencian que los productos de la migración y la globalización no son homogéneos, sino que despierta múltiples maneras de imaginar la pertenencia, construyendo una base identitaria coproducida (por el grupo migrante y el de origen) a distancia. (Yúdice, s/a, 109)

A partir de estas rupturas en el orden o momentos de crisis, o como prefiero llamarlo, tensiones estructurales en las sociedades, se hace necesario analizar las reconfiguraciones que se desarrollan sobre los sentidos de pertenencia social. Es decir, ayudándonos de la noción de diáspora, tratar de analizar y dar cuenta de la manera en que se construye la identidad de un nosotros, cuando ésta está atravesada por la distancia geofísica, aunque muchas veces acortada por los medios masivos de comunicación y las tecnologías multimedia.

En conclusión, puedo decir que las elaboraciones teóricas sobre diásporas dan para pensar muchas realidades de desplazamiento poblacional, específicamente las identidades de personas que se han movilizado de sus lugares de origen aunque no hayan traspasado el ámbito nacional ni siquiera estatal. Además, algunas de sus posturas bajo esquemas epistemológicos posmodernistas, complejos y multidisciplinarios son de gran utilidad para analizar realidades sociales contemporáneas, en general, y las identidades (en tanto fenómeno social), en particular. 


\section{Notas}

1 En este momento me encuentro estudiando el doctorado en Ciencias Sociales y Humanísticas en el Centro de Estudios Superiores de México y Centroamérica (CESMECA) de la UNICACH. El proyecto de investigación que estoy trabajando se denomina "Reconstrucciones Identitarias en el Espacio Universitario. De la reflexión a la acción”. Únicamente señalo la investigación como contexto en el que han surgido las reflexiones aquí vertidas, dejando con esto claro que este artículo no contiene un análisis que se desprenda de la producción de resultados de esta investigación, sino que se centra en el debate teórico desde categorías y teorías que han sido revisadas para la elaboración de la propuesta.

${ }^{2} \mathrm{La}$ UNICH es una propuesta educativa de reciente creación que emerge bajo la preocupación de la exclusión histórica que se ha dado a los diferentes grupos étnicos coexistentes en nuestro país, es decir, se construye desde la preocupación por la inclusión de los grupos indígenas en los espacios de educación superior. Además, se sustenta bajo un modelo educativo con enfoque intercultural, que plantea tres principios básicos para su operación: El reconocimiento al saber ancestral de los pueblos originarios; la transformación de la visión de la cultura hegemónica; y, por consiguiente, el reconocimiento de la diversidad de las tradiciones indígenas (Casillas y Santini, 2006 y 2009).

${ }^{3}$ Por supuesto, no descarto la posibilidad de que los jóvenes de la UNICH puedan verse y pensarse a sí mismos como grupo o como comunidad, pero por ahora no puedo afirmarlo en tanto que me falta trabajo de campo y análisis de materiales como para poder hacerlo.

${ }^{4}$ Es importante comentar que no todos los autores que han trabajado con la noción de diáspora parten de conceptualizarla como grupo, comunidad o entidad, como expondré más adelante. Sin embargo, la aclaración que hago aquí con relación a los estudiantes, tiene que ver, por un lado, con la necesidad ubicar que mis sujetos de estudio no están siendo pensados como grupo, y, por el otro, con la pretensión de tomar distancia con las posturas en que las diásporas son pensadas como grupos.

${ }^{5}$ En este caso remito a la cultura de origen, de manera diferenciada de la comunidad de origen, pues resulta que muchos jóvenes no desean hacer sus investigaciones en sus comunidades de origen, pero sí en alguna comunidad indígena que les permita conocer la cultura de la que forman parte.

${ }^{6}$ Es muy interesante en este sentido el análisis que hace Hall (1999) al estudiar la diáspora de los caribeños como identidad cultural. Es importante señalar que él menciona la eficacia simbólica de la postura esencialista para explicar la diáspora como un hecho de reunificación imaginaria que parte de las prácticas de representación (postura nominalista) de dicho grupo. Por ello puede decirse que su postura analítica, epistemológica y ontológica de la identidad parte de la propuesta constructivista.

7 "Identity is, therefore, a vital component of diasporas; it transforms them from the physical reality of dispersal into the psychisocial reality of diaspora" (Butler, 2001: 207).

${ }^{8}$ Es importante destacar que conservo el término de sociedad mayoritaria únicamente por ser la noción que ha servido para caracterizar a las sociedades que son originarias de los lugares de residencia de las poblaciones que se han desplazado, pero que en la actualidad no necesariamente es el número o la cantidad de población la que fundamenta esta forma de nombrarla.

9 Cuando Cohen (1996: 517) habla de sincretizadas básicamente está haciendo referencia a identidades mezcladas, creadas o recreadas de diferentes maneras y retomando las particularidades culturales, que poco consideran en la reconstitución identitaria $-\mathrm{y}$ si lo hacen es de manera fraccionada porque les resulta indiferente- los elementos de los estados-nación.

${ }^{10}$ Una práctica de residencia distinta que podemos observar en los jóvenes tiene que ver con las prácticas 
sexuales que se ven transformadas considerablemente, pues en la mayoría de las comunidades el noviazgo con contacto sexual visible o los múltiples noviazgos previos al matrimonio no son elementos usuales de los esquemas de significación. Sin embargo, durante su residencia lejos del hogar esta es una práctica que en la mayoría de los jóvenes se ve transformada.

"l Entiéndase por representación a la construcción imaginaria que se genera a través del proceso de identificación que permite al sujeto o colectivo ubicarse o posicionarse, es decir, enunciar (nombrar) sus posiciones.

${ }^{12}$ Por el momento no puedo profundizar más en estas diversidades puesto que aún no he desarrollado el trabajo de campo. Empero, estas observaciones son producto de la convivencia con ellos y de pláticas que he tenido la oportunidad de entablar con muchos jóvenes universitarios.

\section{Bibliografía}

Adamson, F., y Demetriou, M. (2007), "Remapping the Boundaries of 'State' and 'National Identity': Incorporating Diasporas into IR Theorizing", en European Journal of International Relations, vol. 13 (4), pp. 489-526.

Akzin, B. (1964), “Estado y Nación”, 1968, Brevarios 200, México: FCE.

Alcoff, L. (1989), "Feminismo cultural versus posestructuralismo: la crisis de identidad en la teoría femenina", en Feminaria, Año II, núm. 4, noviembre de 1989, Buenos Aires:Tejepalabras, pp. 1-18.

Anderson, B.. (1983), "Comunidades Imaginadas" (tomado de Imagined Comunities. Reflections on the Origin and Spread of Nationalism, décima edición, Londres: Verso Editions and NLB, pp. 2-7), en Giménez, Gilberto, 2005, Teoría y análisis de la cultura, vol. II, México: CONACULTA, pp. 88-93.

Anderson, B. (1998), "Nationalism, Identity, and the Logic of Serialty", en The Spectres of comparison. Nacionalismo, Southeast Asia and The World, Verso, Londres, pp. 29-45.
Barth, F. (Comp.) (1969), Los grupos étnicos y sus fronteras. La organización social de las diferencias culturales, México: FCE, 1976.

Bourdieu, P. (1980), "Estructuras, habitus, prácticas", en El sentido práctico, Madrid: Taurus, 1991, pp. 91-111.

Braziel, J. y Mannur, A. (2003), "Nation, Migration, Globalization: Poinst of Contention in Diaspora Studies", en Braziel, R. y Mannur, A. (eds.), Theorizing Diaspora, Londres: Blackwell, pp. 1-22.

Brubaker, R. (2005), "The 'diaspora' diaspora”, en Ethnic and Racial Studies, vol. 28, núm. 1, pp. 1-19. [consultado el 3 de agosto de 2009, en http://dx.doi.org/10.1080/0 141987042000289997.

Butler, K. (2001), "Defining Diaspora, Redefining Discourse", en Diaspora. A journal of Transnational Studies, vol. 10, núm. 2, ‘pp. 189-220.

Casillas M., M. y L. Santini V. (2006), Universidad Intercultural. Modelo educativo, en Serie Documentos, México: CGEI-SEP.

Casillas M., M. y L. Santini V. (2009), Universidad Intercultural. Modelo educativo, en Serie Documentos, 2da. edición, México: CGEIB-SEP.

Clifford, James (1997), "Las diásporas", en: Itinerarios Transculturales, Barcelona: Gedisa, pp. 299-340.

Cohen, R. (1996), "Diasporas and the Nation-State: from Victims to Challengers, en International Affairs, vol. 72, núm. 3, Ethnicity and International Relations, pp. 507-520, [consultado el 5 de agosto de 2009, en http://links.jstor.org/sici?sici=0020\%2972\%3A3\%3C507\%3ADATNFV\%3E2.0.CO\%3B2-X .

Dubar, C. (2002), La crisis de las identidades: la interpretación de una mutación, Barcelona: Bellaterra..

D’Andrea, D. (2005), "Las razones de la etnicidad entre la globalización y el eclipse de la política" (tomado de Identitá e conflitti, Franco Angeli, Milán, pp. 83-91), en Giménez, Gilberto, Teoría y análisis de la cultura, vol. II, México: CONACULTA, pp. 61-71.

Fábregas PUIG, A. (1984), "Desde el sur una revisión del CONCEPTO DE FRONTERA”, en Ensayos Antropológicos 1990-1997, México: UNICACH, pp. 107-114. 
García-Canclini, N. (2000), "Escenas sin territorio: Cultura de los migrantes e identidades en transición", en Valenzuela A., J. M. (Coord.), Decadencia y auge de las identidades: cultura nacional, identidad cultural y modernización, México El Colegio de la Frontera Norte, segunda edición, pp. 191-208.

Giménez, G. (2000), "Materiales para una teoría de las identidades sociales", en Valenzuela A., J. M. (coord.), Decadencia y auge de las identidades: cultura nacional, identidad cultural y modernización, México: El Colegio de la Frontera Norte, segunda edición, pp. 45-78.

Giménez, G. (2005I), Teoría y análisis de la cultura, vol. I, México: CONACUlTA-ICOCUlT, Colección Intersecciones.

Giménez, G. (2005II), Teoría y análisis de la cultura, vol. II, México: CONACULTA-ICOCUlT, Colección Intersecciones.

Hall, S. (1996), "Introducción: ¿Quién necesita 'identidad'?", en Hall, S. y Du Gay, P., (comps.), Cuestiones de identidad cultural, Buenos Aires: Amorrortu, pp. 13-39.

Hall, S. (1999), "Identidad cultural y diáspora”, en Castro G., S. et.al., Pensar (en) los intersticios: Teoría y práctica de la crítica poscolonial, Bogotá: Ceja, pp. 131-145.

Habermas, J. (1996), "El estado nacional europeo. Sobre el pasado y el futuro de la soberanía y de la ciudadanía", en La inclusión del otro. Estudios de teoría política, 1999, Barcelona: Paidós Básica, núm. 98, pp. 81-105.

Hobsbawn, E. (1995), Historia del Siglo XX, Barcelona: Crítica.

Mitre Fernández, E., et.al.(1997), “La cristiandad Medieval y las Formulaciones Fronterizas", en: Fronteras y
Fronterizos en la historia, España: Universidad de Valladolid, pp. 9-52.

Pintor V., A. (2000), "Modernización, escuela e identidad en América Latina", en: Investigación y Desarrollo, núm.ll, Colombia: Universidad del Norte Barranquilla, pp. 106-121,.[18 de Junio de 2009], en http://redalyc.uaemex.mx/redalyc/src/inicio/ ArtPdfRed.jsp?iCve=26801106.

Riquer F., F. (1992), "Identidad Femenina en la frontera entre la conciencia y la interacción social”, en Tarrés, M. L., La voluntad de ser. Mujeres en los Noventa, México: El Colegio de México, pp. 51-64.

Sayad, A. (2009), "Estado, nación e inmigración", en Apuntes de investigación del CECYP, núm. 13, pp. 101-116.

Schnapper, D. (1999), "From the Nation-State to the Transnational World: On the meaning and Usefulness of Diaspora", en Diaspora. A journal of Transnational Studies, 8, 3, pp. 225-255.

Schutz, A. (1974), "La vuelta al hogar", en Estudios de teoría social, Buenos Aires: Amorrortu.

Smith, A. (1991), "Tres conceptos de nación”, en Revista de Occidente, núm. 161, pp. 7-22.

Tölölyan, K. (1996), "Rethinking Diaspora(s): Stateless Power in the Transnational Moment", en Diaspora. A journal of Transnational Studies, 5, 1, pp. 3-35.

Wimmer, A. y Glick Schiller, N. (2002), "Methodological Nacionalism and Beyond: Nation-State Building, Migration and the Social Sciences", en Global Networks, 2, 4, pp. 301-334.

Yúdice, G., s/a, "iUna o varias identidades? Cultura, globalización y migraciones", en Nueva Sociedad, núm. 201, pp. 106-116. 